\title{
Oscillation Patterns of SX Phœnicis Variables in Globular Clusters
}

\author{
J. O. Petersen ${ }^{1}$, M. Quaade ${ }^{1}$, M. I. Andersen ${ }^{2}$, L. M. Freyhammer ${ }^{3}$
}

Abstract. Based on new analyses of light curves of SX Phœnicis variables in globular clusters and on results taken from the literature, an overview of oscillation patterns in this subgroup of $\delta$ Scuti variables is given. Belonging to the Blue Straggler stars, they are expected to have a more complicated life history than "normal" $\delta$ Scuti stars in the field or in open clusters. The overall picture is that the patterns found in this SX Phe group seem to be similar to the patterns that are well established for standard field $\delta$ Sct stars.

\section{Introduction}

With the purpose of characterizing the observed pulsation properties of SX Phe stars in globular clusters we have collected data from the literature and performed new pulsation analyses of light curves published as time series in several data bases. In section 2 we present the resulting statistics, give a rough description of basic pulsation properties and compare oscillation patterns with those of field $\delta$ Scuti variables.

\section{Observed Pulsation Properties and Oscillation Patterns}

Table 1 presents an overview of oscillation properties of SX Phe stars in ten globular clusters. See Petersen et al. (2000) for references to the literature and time series. From a physical point of view the traditional introduction of the high-amplitude $\delta$ Sct (HADS) group (with $\Delta V>0.3 \mathrm{mag}$ ) seems artificial. However, for discussion of oscillation patterns this distinction is very convenient because HADS oscillate mainly in one or two modes only, whereas low-amplitude $\delta$ Sct stars usually have a complicated oscillation pattern. Column 4 of Table 1 gives the number of mono-mode stars (MM), here defined as all stars where only one oscillation frequency is known. Of course, with more accurate observations most of these variables will turn out to have a more complicated oscillation spectrum. Besides the number of well established, or suspected double-mode

\footnotetext{
${ }^{1}$ Niels Bohr Institute for Astronomy, Physics and Geophysics, Astronomical Observatory, Juliane Maries Vej 30, DK-2100 Copenhagen $\varnothing$, Denmark

${ }^{2}$ Division of Astronomy, University of Oulu, P.O. Box 3000, FIN-90401 Oulu, Finland

${ }^{3}$ University of Brussels, Pleinlaan 2, B-1050 Brussels, Belgium and Nordic Optical Telescope, Apartado 474, E-38700 Santa Cruz de La Palma, Spain
} 
stars (DM), we also give the number of stars with close modes (CM), here meaning that the observed frequencies are too close to be understood as loworder radial modes. The correct physical interpretation is unknown at present.

Table 1. Known SX Phe stars in globular clusters.

\begin{tabular}{|c|c|c|c|c|c|c|c|}
\hline \multirow{2}{*}{$\begin{array}{l}\text { Name of } \\
\text { cluster }\end{array}$} & \multicolumn{5}{|c|}{ No. of SX Phe stars } & \multirow{2}{*}{$\begin{array}{l}\text { Period interval } \\
{[d]}\end{array}$} & \multirow{2}{*}{$\begin{array}{l}{[\mathrm{Fe} / \mathrm{H}]} \\
{[\mathrm{dex}]}\end{array}$} \\
\hline & All & HADS & $\overline{\mathrm{MM}}$ & $\overline{\mathrm{DM}}$ & CM & & \\
\hline NGC 5053 & 5 & 0 & 4 & $0 / 1$ & 0 & $0.0342-0.0393$ & -2.29 \\
\hline NGC 5466 & 6 & 3 & $6:$ & $0 / 0$ & 0 & $0.040-0.055$ & -2.22 \\
\hline NGC 4372 & 8 & 6 & 8: & $0 / 0$ & 0 & $0.041-0.067$ & -2.09 \\
\hline NGC 6397 & 2 & 0 & 1 & $0 / 1$ & 0 & $0.030-0.038$ & -1.95 \\
\hline Ruprecht 106 & 3 & 1 & 3: & $0 / 0$ & 0 & $0.0395-0.0490$ & -1.80 \\
\hline$\omega$ Cen & 33 & 5 & 24 & $3 / 3$ & 3 & $0.0339-0.0656$ & -1.62 \\
\hline M3 & 3 & 1 & 3: & $0 / 0$ & 0 & $0.0372-0.0403$ & -1.57 \\
\hline NGC 288 & 6 & 3 & 4 & $1 / 0$ & 1 & $0.039-0.079$ & -1.24 \\
\hline 47 Tuc & 6 & 0 & 0 & $2 / 3$ & 1 & $0.055-0.102$ & -0.76 \\
\hline M71 & 1 & 0 & 1: & $0 / 0$ & 0 & 0.050 & -0.73 \\
\hline Total & 73 & 19 & 54 & $6 / 8$ & 5 & & \\
\hline
\end{tabular}

Let us very roughly divide the field $\delta$ Sct variables into classes according to the schematic oscillation types used in Table 1 for the SX Phe stars in globular clusters. About $10 \%$ are HADS. According to Table 1 the HADS fraction in the globular cluster variables is considerably higher, about $25 \%$. However, this is probably due to the fact that the data available for globular clusters today are insufficient to detect many low-amplitude variables. In the majority of cases only one oscillation period is known. Thus, most field stars also belong to the mono-mode class, when this class is defined precisely as in Table 1 . We estimate six stars as reliable double-mode variables, eight stars as suspected double-mode stars, and five with close frequencies out of 73 SX Phe stars. Again, the rather poor statistics do not allow us to claim a significant difference between this sample and the field variables.

Our main conclusion is that the SX Phe stars in globular clusters seem to show the oscillation patterns that are well-known from field $\delta$ Sct variables. The presently available data are not sufficient to give a reliable, more-detailed comparison of the occurrence of the different main pulsation types between the variables in globular clusters and the field. Within the uncertainties the data are in agreement with the simplest assumption that the overall oscillation patterns excited are the same in the two cases.

\section{Reference}

Petersen, J. O., Quaade, M., Freyhammer, L. M., \& Andersen, M. I. 2000, in ASP Conf. Ser., 6th Vienna Workshop in Astrophysics: $\delta$ Scuti and Related Stars, ed. M. Breger \& M. Montgomery (San Francisco: ASP), in press 